\title{
Antiphospholipid antibody syndrome presenting with disseminated bleeding and spinal subdural hemorrhage
}

\author{
NV Jayachandran*,1, L Rajasekhar ${ }^{1}$, G Narsimulu ${ }^{1}$ and V Prasad $^{2}$ \\ ${ }^{1}$ Department of Rheumatology, Nizam's Institute of Medical Sciences, Hyderabad, Andhra Pradesh, India; ${ }^{2}$ Department \\ of Neurosurgery, Nizam's Institute of Medical Sciences, Hyderabad, Andhra Pradesh, India
}

Study design: Case report.

Objectives: To report a rare cause of spinal subdural hematoma.

Setting: A tertiary care university hospital in Andhra Pradesh, India.

Methods: Detailed evaluation and reporting of a case admitted in the hospital.

Results: A case of antiphospholipid antibody syndrome (APS) was found to present with spontaneous spinal subdural hematoma and paraplegia. This is a very rare presentation of APS. Conclusion: This article describes a rare case of APS presenting with disseminated bleeding and spontaneous spinal subdural hematoma, resulting in paraplegia.

Sponsorship: Nil.

Spinal Cord (2007) 45, 753-755; doi:10.1038/sj.sc.3102041; published online 6 February 2007

Keywords: systematic lupus erythematosus antiphospholipid antibody; spontaneous spinal subdural hematoma; paraplegia

\section{Introduction}

Antiphospholipid antibody syndrome (APS) also known as anticardiolipin antibody syndrome, lupus anticoagulant syndrome, or Hughes syndrome was first described by Hughes et $a l^{1}$ as a condition characterized by propensity for arterial/venous thrombosis, recurrent fetal wastage and thrombocytopenia. The presence of an antiphospholipid antibody can be documented by a solid phase serum assay of anticardiolipin, or an inhibitor of phospholipid-dependent clotting (lupus anticoagulant) or both. ${ }^{2}$

APS can occur as an isolated diagnosis, known as primary antiphospholipid antibody syndrome (PAPS) or can be associated with systemic lupus erythematosus (SLE) or other rheumatic diseases when it is known as secondary APS. ${ }^{3}$ Even though APS is a condition commonly associated with arterial or venous thrombosis, very rarely it can lead on to hemorrhage also. ${ }^{4}$ This occurs when patients with APS develop antibodies directed against prothrombin leading on to clinically significant hypoprothrombinemia leading to hemorrhage. There are only very few case reports of lupus anticoagulant-hypoprothrombinemia syndromes. Spontaneous spinal subdural hematoma is unusual and difficult to treat. Herein, we report a case of SLE with

*Correspondence: NV Jayachandran, Department of Rheumatology, Nizam's Institute of Medical Sciences, Panjagutta, Hyderabad, Andhra Pradesh, India secondary APS who presented with disseminated bleeding and acute onset paraplegia due to spinal subdural hematoma.

\section{Case report}

Materials and methods

A 55-year-old lady presented to us with history of acute onset lower thoracic pain followed by weakness of both lower limbs of 1 day duration. At the time of admission she had bleeding per rectum also. She was catheterized as she had urinary retention and upon catheterization it was found that she was having hematuria. The hematuria was massive and persistent.

She gave past history of polyarthritis of 6 years duration. Six months prior to admission she was admitted in the same hospital with fever and breathlessness. Evaluation at that time showed that she had SLE with secondary APS. The relevant investigations are shown in Table 1.

At that time she also had left pleural effusion, right perinephric collection, and anemia. An ultrasoundguided aspiration from perinephric collection was attempted and obtained $2 \mathrm{ml}$ of altered blood. Her platelet count was near normal and was not on any anticoagulants at that time. She was also a diabetic. She also gave history of one spontaneous abortion in the second month of pregnancy. 
Table 1 Investigations

\begin{tabular}{|c|c|c|}
\hline Investigation & $\begin{array}{l}\text { Patient's } \\
\text { value }\end{array}$ & Reference \\
\hline ANA & Positive & Homogenous pattern \\
\hline AntidsDNA & $158 \mathrm{IU} / \mathrm{ml}$ & Positive $>40 \mathrm{IU} / \mathrm{ml}$ \\
\hline ACL IgG & $70 \mathrm{GPL}$ & $\begin{array}{l}\text { Positive }>10 \mathrm{GPL} \\
\text { units } / \mathrm{ml}\end{array}$ \\
\hline ACL IgM & $130 \mathrm{MPL}$ & $\begin{array}{l}\text { Positive }>7 \mathrm{MPL} \\
\text { units/ml }\end{array}$ \\
\hline PT (initial admission) & $16.5 \mathrm{~s}$ & Control $12 \mathrm{~s}$ \\
\hline APTT (initial admission) & $38.2 \mathrm{~s}$ & Control $30 \mathrm{~s}$ \\
\hline PT (present admission) & $13 \mathrm{~s}$ & Control $12 \mathrm{~s}$ \\
\hline $\begin{array}{l}\text { APTT (present } \\
\text { admission) }\end{array}$ & $45.9 \mathrm{~s}$ & Control $30 \mathrm{~s}$ \\
\hline Platelet & $\begin{array}{l}120000 / \\
\mathrm{cmm}\end{array}$ & $1.5-4 \mathrm{lakh} / \mathrm{cmm}$ \\
\hline Fibrinogen & $560 \mathrm{mg} / \mathrm{dl}$ & Normal $200-400 \mathrm{mg} / \mathrm{dl}$ \\
\hline Factor VIII & $>150 \%$ & Reference $60-150 \%$ \\
\hline Factor IX & $>150 \%$ & Reference $60-150 \%$ \\
\hline
\end{tabular}

ANA, antinuclear antibody; AntidsDNA, anti-doublestranded DNA antibody; ACL IgG, anticardiolipin antibody (immunoglobulin G type); ACL IgM, anticardiolipin antibody (immunoglobulin $\mathrm{M}$ type); PT, prothrombin time; APTT, activated partial thromboplastin time

At the time of present admission, she was paraplegic with a sensory level at thoracic spine T10. Her spine and skull were within normal limits. She had no cranial nerve palsies. She had grade zero power below T10. Ultrasonography of the bladder showed irregular hypoechoic lesion along right posterolateral wall of bladder suggestive of a clot.

A magnetic resonance imaging of the thoracic spine showed a nonenhancing intradural lesion from T8 to T12 level displacing the cord to the left and compressing it (Figures 1 and 2). She was treated with fresh frozen plasma and corticosteroids and was taken up for surgical decompression.

\section{Operative findings}

After laminectomy, dural sac was seen discolored and nonpulsatile. Once dura was opened, an organized clot was seen with partial liquefaction. This clot was gently dissected off the spinal cord and evacuated by suction and bipolar coagulation. Thorough irrigation removed the remnants of clot. The saline-filled dural sac started pulsating slowly. Dural incision was closed and wound approximated in layers. The postoperative period was uneventful. Her high blood sugar was controlled with insulin. Her hematuria spontaneously subsided and a repeat Ultrasonography of bladder was within normal limits. At 3 months follow-up she had 10\% improvement in her motor power.

\section{Discussion}

APS is a syndrome complex characterized by recurrent venous/arterial thrombosis, obstetrical complications

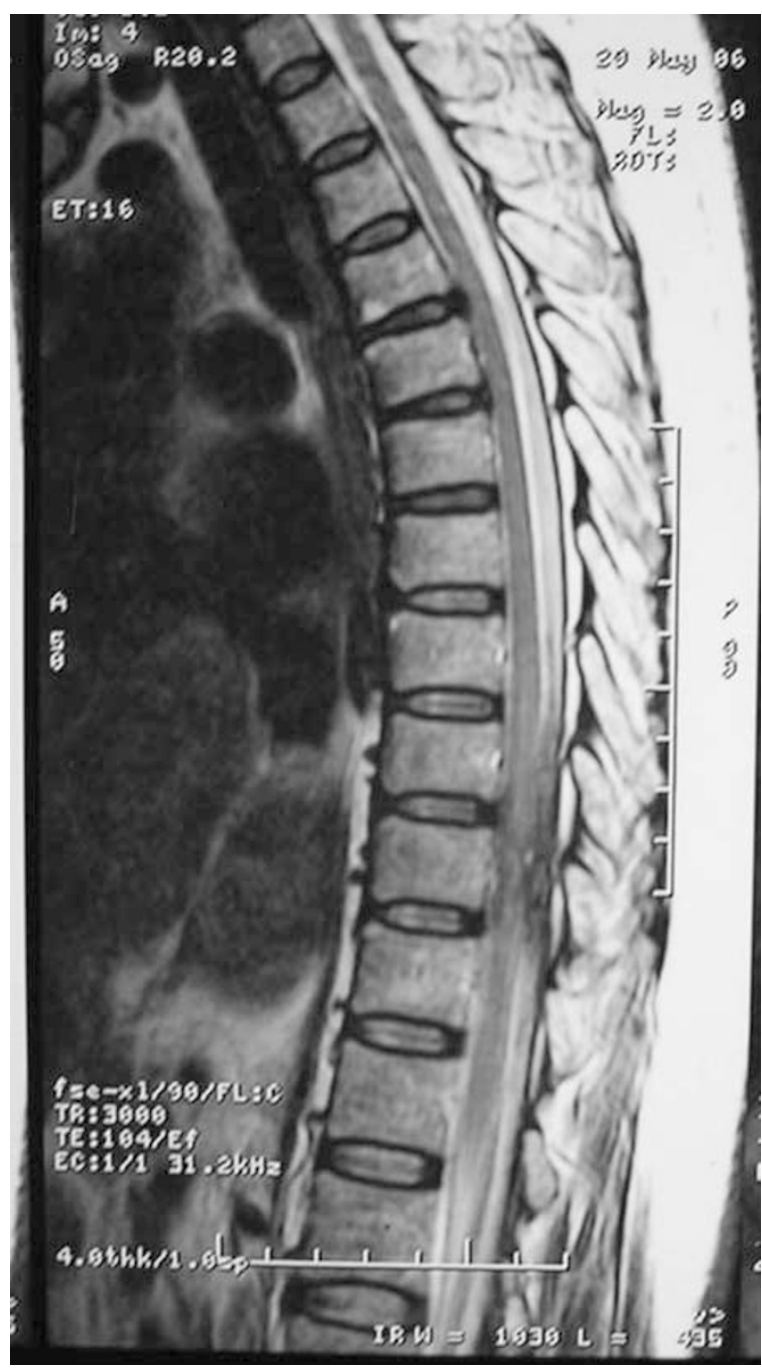

Figure 1 Sagittal T2 weighted MR images showing iso/ hypointense intradural extramedullary collection at the level of T10 displacing the spinal cord

like recurrent fetal loss, intrauterine growth retardation, and thrombocytopenia. It can occur as a primary disease without any underlying connective tissue disease when it is known as primary APS, and also as a complication secondary to SLE or lupus like syndromes.

Our patient had history of nonerosive chronic poly arthritis, serositis, positive antinuclear antibody, positive anti-double-stranded DNA, thereby satisfying the American College of Rheumatology criteria for the diagnosis of SLE.

This patient also had prolonged activated partial thromboplastin time during the present and previous admissions, which were at an interval of 6 months. She also had elevated levels of serum IgG and IgM anticardiolipin antibodies. These features suggest that the patient had associated secondary APS also. She developed an acute paraplegia without any history of trauma, and at that time she also developed spontaneous bleeding per rectum and also severe hematuria, thereby 


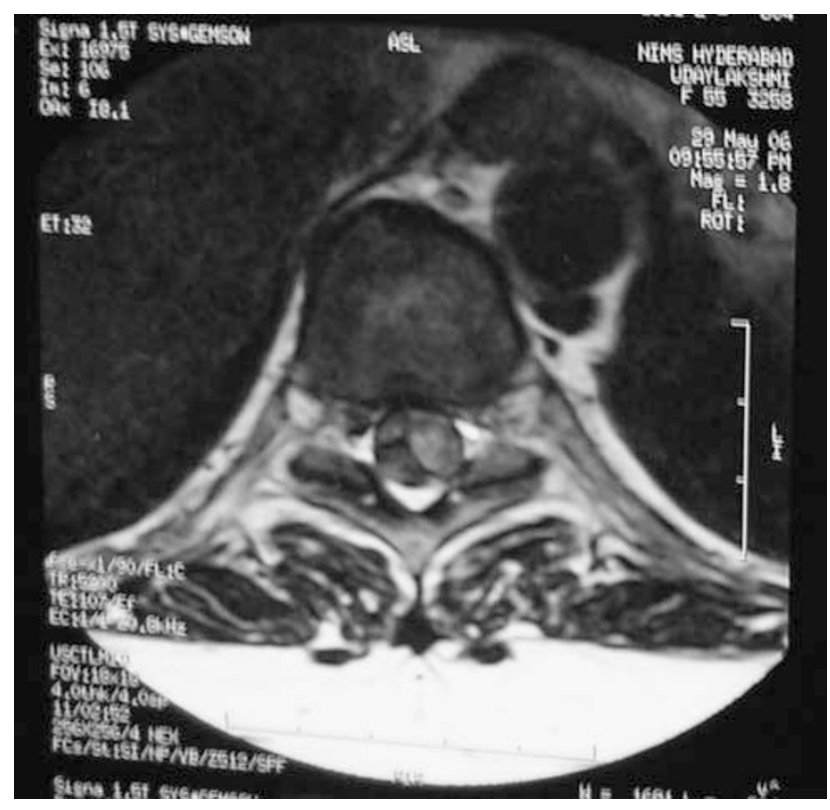

Figure 2 Axial T2 weighted MR images showing iso/ hypointense intradural extramedullary collection at the level of T10 displacing the spinal cord

suggesting a state of disseminated bleeding. The spinal subdural hemorrhage was confirmed by surgery.

APS is usually characterized by thrombosis. But patients with the lupus anticoagulant directed against prothrombin, on rare occasions, may develop clinically important hypoprothrombinemia leading to hemorrhage. This is a very rare complication of APS and has previously been reported to occur more often in children. ${ }^{5}$

Spinal subdural hematoma is rare and a few cases only have been reported. It is usually diagnosed at operation or postmortem examination because it lacks a typical clinical or radiological feature. First report on spinal subdural hematoma was made by Schiller et $a l^{6}$ in a hemophilic patient. In a survey of 58 cases of spinal subdural hematoma, females were more often affected and the age ranged from 4 months to 80 years. ${ }^{7}$ The hematomas were most commonly found in the sixth decade and most often at the thoracic and thoracolumbar regions. Coagulation abnormalities secondary to blood dyscrasias or anticoagulant therapy were responsible for hemorrhage in majority of cases $(36.8 \%)$. Severe back pain followed by radicular pain in the extremities was the initial complaint in most of the case. Paraplegia was noted in $70 \%$ of cases preoperatively.

Spinal subdural hematoma in relation to APS has never been reported in the literature.

\section{Acknowledgements}

We gratefully acknowledge the help of Dr Pradeep Kumar Shenoy Chandrasekhara and Dr P Arun kumar for their help in managing this patient.

\section{References}

1 Hughes GRV. Thrombosis, abortion, cerebral disease and lupus anticoagulant. BMJ 1983; 287: 1088-1089.

2 Hughes GRV. The Antiphospholipid antibody syndrome: ten years on. Lancet 1993; 342: 341-344.

3 Bick RL, Baker WF. The Antiphospholipid and thrombosis syndromes. Med Clin North Am 1994; 78: 667-684.

4 Erkan D, Bateman H, Lockshin MD. Lupus anticoagulant hypoprothrombinemia syndrome associated with SLE: report of two cases and review of literature. Lupus 1999; 8: $560-564$.

5 Becton DL, Stine KC. Transient lupus anticoagulants associated with hemorrhage rather than thrombosis: the hemorrhagic lupus anticoagulant syndrome. J Pediatr 1997; 130: $998-1000$.

6 Schiller F, Neligan G, Budtz-Olsen O. Surgery in hemophilia: a case of spinal subdural hematoma producing paraplegia. Lancet 1948; 27: 842-845.

7 Russel N, Benvit BG. Spinal subdural hematoma, a review. Surg Neurol 1983; 20: 133-137. 